\title{
Skin Thickness of the Anterior, Anteromedial, and Anterolateral Thigh: A Cadaveric Study for Split- Skin Graft Donor Sites
}

\author{
Jeffrey CY Chan ${ }^{1}$, John Ward ${ }^{1}$, Fabio Quondamatteo ${ }^{2}$, Peter Dockery ${ }^{2}$, John L Kelly ${ }^{1}$ \\ ${ }^{1}$ Department of Plastic and Reconstructive Surgery, Galway University Hospital, Galway; ${ }^{2}$ Department of Anatomy, National University of \\ Ireland, Galway, Ireland
}

Background The depth of graft harvest and the residual dermis available for reepithelization primarily influence the healing of split-skin graft donor sites. When the thigh region is chosen, the authors hypothesize based on thickness measurements that the anterolateral region is the optimal donor site.

Methods Full-thickness skin specimens were sampled from the anteromedial, anterior, and anterolateral regions of human cadavers. Skin specimens were cut perpendicularly with a custom-made precision apparatus to avoid the overestimation of thickness measurements. The combined epidermal and dermal thicknesses (overall skin thickness) were measured using a digital calliper. The specimens were histologically stained to visualize their basement membrane, and microscopy images were captured. Since the epidermal thickness varies across the specimen, a stereological method was used to eliminate observer bias.

Results Epidermal thickness represented 2.5\% to 9.9\% of the overall skin thickness. There was a significant difference in epidermal thickness from one region to another $(P<0.05)$. The anterolateral thigh region had the most consistent and highest mean epidermal thickness $(60 \pm 3.2 \mu \mathrm{m})$. We observed that overall skin thickness increased laterally from the anteromedial region to the anterior and anterolateral regions of the thigh. The overall skin thickness measured $1,032 \pm 435 \mu \mathrm{m}$ in the anteromedial region compared to $1,220 \pm 257 \mu \mathrm{m}$ in the anterolateral region.

Conclusions Based on skin thickness measurements, the anterolateral thigh had the thickest epidermal and dermal layers. We suggest that the anterolateral thigh region is the optimal donor site for split-skin graft harvests from the thigh.

Keywords Skin surgery / Skin / Photomicrography / Cadaver / Histology
Correspondence: Jeffrey CY Chan Department of Plastic and Reconstructive Surgery, Galway University Hospital, Newcastle Road, Galway, Ireland

Tel: +353-91-524222

Fax: +353-91-524222

E-mail: chancy@eircom.net

The authors acknowledge Mr. John Fury, Senior Anatomy Technician from the Department of Anatomy, National University of Ireland, Galway for his kind support and assistance throughout this study. The authors also acknowledge the donors to the School of Medicine (Department of Anatomy), National University of Ireland, Galway for making this study possible.

No potential conflict of interest relevant to this article was reported.

\section{INTRODUCTION}

Split-skin grafts can be harvested from any part of the body using a hand-held or power-driven dermatome. A split-skin graft provides a simple method of wound closure when primary closure is not possible or healing by secondary intention is undesired or prolonged. Common donor sites include the lumbar region, buttock, thigh, and arm. The ideal donor site characteris- 
tics are highlighted in Table 1. In clinical practice, when the defect size is small to moderate, the thigh is frequently chosen as the donor site of choice because: 1) it has the largest surface area of any limb, 2) its location provides easy access and setup for graft harvesting when the patient is in a supine position, 3 ) its firm underlying structures provide a stable platform for the dermatome, and 4) the resultant scar is usually minimal and can be easily hidden under clothing.

However, delayed donor site healing is a frequently encountered complication. Cosmetically unacceptable hypertrophic scarring and pigmentation changes have also been described $[1,2]$. Consequently, numerous studies have attempted to determine which dressing is optimal to expedite donor site healing. Although some studies have recommended specific dressings $[3,4]$, others have reported no difference in healing times $[5,6]$. Interestingly, Giele et al. [7] observed no difference between the commonly used calcium alginate dressings and adhesive retention dressings [8]. Grafting the donor site to accelerate healing has been recommended [9], while some advocate the scalp as the donor site of choice $[10,11]$. The scalp has been shown to heal more quickly than other areas but the head must be shaved [10]. However, complications such as folliculitis, alopecia, and blood loss can occur on the scalp $[10,12]$. Nevertheless, it has been suggested that the rich blood supply and thick dermis of the scalp lead to rapid healing, independent of the type of dressings used.

The dermis does not regenerate once it is harvested. The epidermal layer, however, reepithelizes through the migration of epidermal cells originating from hair follicles and adnexal structures within the dermis [13]. Hence, donor site healing is primarily influenced by the depth of the graft harvest, which consequently determines the thickness of the residual dermis available for reepithelization. For this reason, it would be intuitive to opt for a donor site with the thickest dermis whenever possible to prevent visible scarring and preserve as much of the remain-

Table 1. Characteristics of the ideal donor site for splitthickness skin grafts

\section{Characteristics}

Ease of access during surgery

Ease of preparation

Ease of harvesting

Minimal bleeding

Minimal pain

Uncomplicated postoperative care

Low risk of infection

Rapid healing

Minimal scarring or pigmentation changes

Minimal disturbance with rehabilitation ing dermal layer as possible.

Since the thigh is routinely used as the preferred donor site, we aimed to establish the optimal region of the thigh for harvesting split-thickness skin grafts. Anecdotal evidence suggests that the anterolateral aspect of the thigh has thicker skin than that in the anteromedial aspect. To our knowledge, no published literature has confirmed this assumption. We hypothesized that the anterolateral thigh region is the optimal donor site based on thickness measurements. Therefore, we aimed to assess variations in skin thickness from the anteromedial, anterior, and anterolateral regions of the thigh and their corresponding epidermal thickness in a cadaveric study. To measure true skin thickness, we designed a novel precision equipment for sectioning skin specimens and applied an unbiased stereological method to determine skin thickness.

\section{METHODS}

Full thickness skin specimens were sampled from the anteromedial, anterior, and anterolateral regions of the thigh of formaldehyde-fixed human cadavers $(n=6)$ from the Department of Anatomy. Unsystematically sectioning the skin specimens introduces inaccuracy in the measured thickness of the specimen. However, slicing the skin specimens accurately at right angles avoids overestimating the thickness during sampling (Fig. 1). To ensure that the true epidermal and dermal thicknesses were sampled, a custom-made precision apparatus was designed and used in this study.

This apparatus consists of a plastic base component and a metal top component that can be secured together (Fig. 2A). The skin specimen is placed between the two components and

\section{Fig. 1. Micrograph of stained skin specimens}

Micrograph demonstrating that unsystematically sectioning the skin specimens at various angles (yellow arrows) introduces inaccuracy into the thickness measurement. Slicing the skin specimens accurately at right angles (red arrows) avoids overestimating the thickness during sampling.

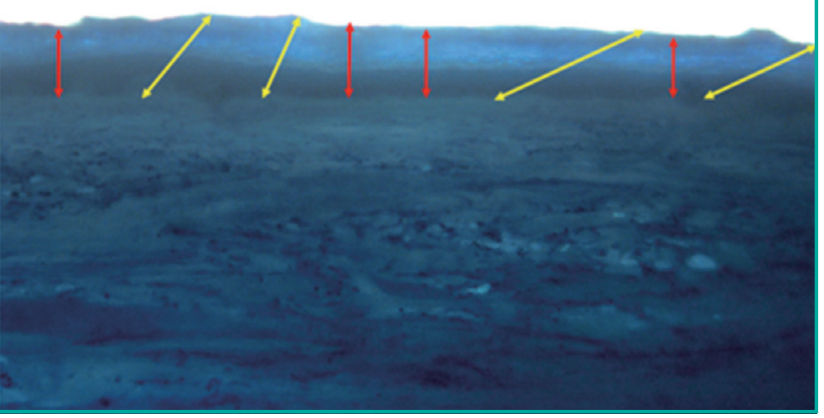


securely locked. The metal component is designed to incorporate parallel slits where scalpel blades can pass at right angles to section the specimen (Fig. 2B). These slits were set at widths of $1 \mathrm{~mm}, 2 \mathrm{~mm}$, or $3 \mathrm{~mm}$.

The combined epidermal and dermal thicknesses were measured using a standard digital calliper. Sectioned skin samples were stained with a dilute solution of haematoxylin and toluidine blue (Fig. 3A). Microscopic images were captured with a digital camera at $10 \times$ magnification (Leica Camera AG, Wetzlar, Germany) (Fig. 3B). Subsequently, the epidermal thickness was determined by employing a stereological method as described by Jensen et al. [14] to overcome observer and measurement bias.
Briefly, a standard grid of $21 \times 11$ points was transposed onto each digital image of the skin section (Fig. 4A) using image analysis software (ImageJ version 1.43, National Institutes of Health, Bethesda, MD, USA). All points where the grid points coincided with the basement membrane on the image were noted. Since the epidermal thickness is variable across the section of skin, the grid provided random points on the image where epidermal thickness can be measured. The epidermal thickness was measured perpendicular to the surface of the epidermis at these pre-determined random points (Fig. 4B). At least six images were taken from each skin section and at least five sections were obtained from each region of each cadaver.

The measurements were entered into a Microsoft Excel spread-

\section{Fig. 2. Custom made skin-sectioning apparatus}

(A) A skin specimen is placed between the plastic base component and the metal top component of a custom-made precision instrument that has predetermined slit thicknesses to allow skin sectioning at a right angle with a scalpel. (B) A 1-mm thick piece of cadaveric skin sectioned at a right angle was obtained.
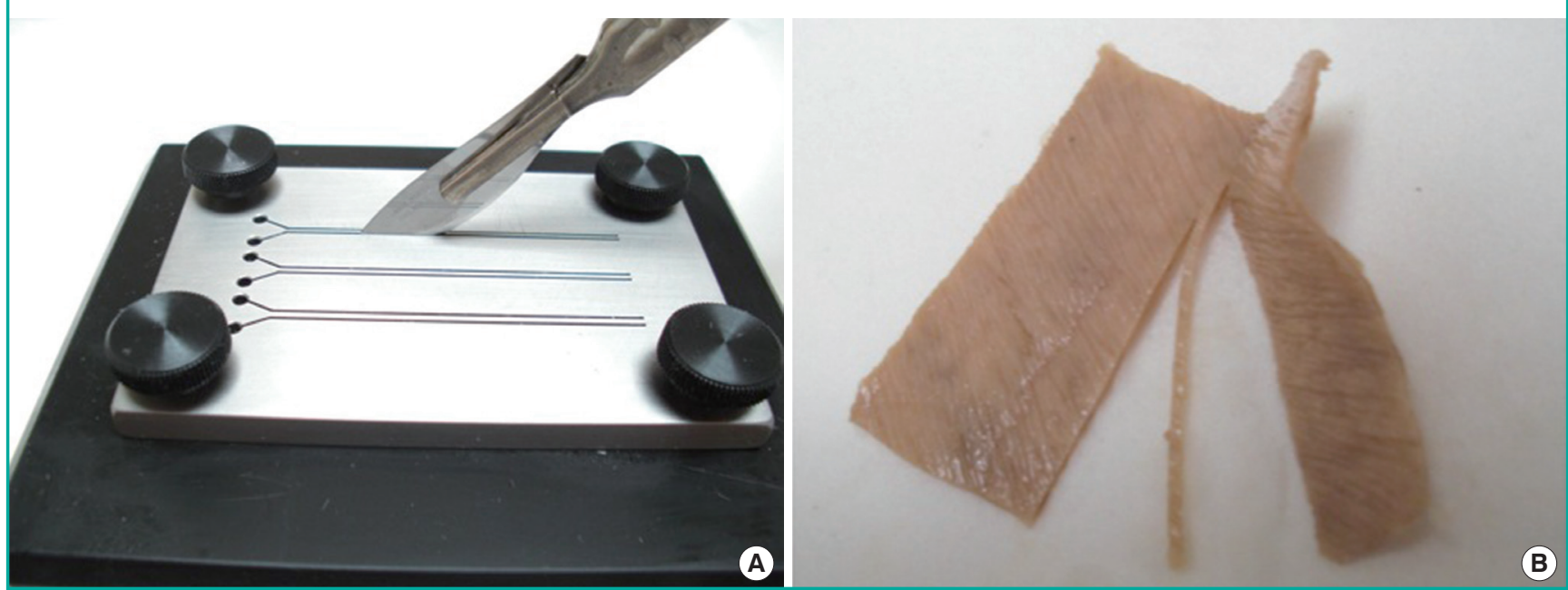

Fig. 3. Sections stained with haematoxylin and toluidine blue

(A) Images of stained skin sections were captured using a microscope mounted with a digital camera at $10 \times$ magnification. (B) Note the variable position of the basement membrane, which results in a variable epidermal thickness.

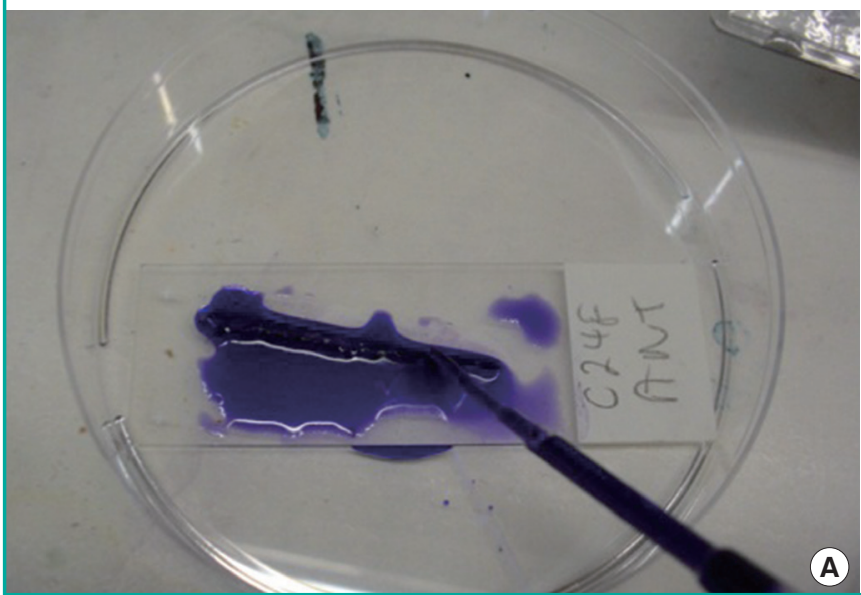




\section{Fig. 4. Stereology method to overcome observer bias}

(A) A uniform grid was transposed on the image (red crosses) and any points that coincided with the basement membrane (highlighted with white crosses) were noted for measurements. (B) The epidermal thicknesses were measured perpendicular to the surface of the epidermis using image analysis software (highlighted with white arrows).

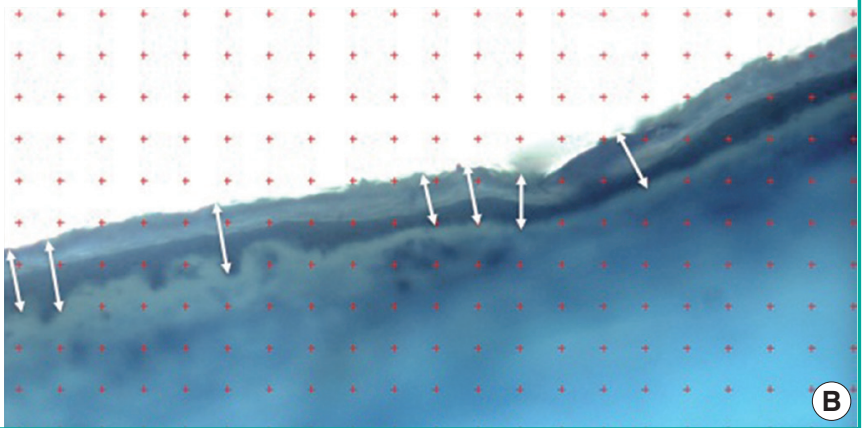

Table 2. Comparison of skin thicknesses for each region

\begin{tabular}{|c|c|c|c|}
\hline Characteristic & $\begin{array}{l}\text { Overall skin thickness } \\
\text { (epidermal and dermal) }\end{array}$ & $\begin{array}{l}\text { Epidermal } \\
\text { thickness }\end{array}$ & $\begin{array}{c}\text { Comparison of } \\
\text { epidermal thickness }\end{array}$ \\
\hline Anteromedial & $1,032 \pm 435$ & $55 \pm 12.5$ & $P<0.05$ \\
\hline Anterior & $1,063 \pm 301$ & $57 \pm 9.4$ & $P<0.05$ \\
\hline Anterolateral & $1,220 \pm 257$ & $60 \pm 3.2$ & Not significant \\
\hline
\end{tabular}

sheet (Microsoft Co., Redmond, WA, USA) and statistical analyses were performed using GraphPad InStat version 3 for Macintosh (GraphPad Software Inc., San Diego, CA, USA). We utilized one-way analysis of variance to test the differences in thickness measurements among the regions and subjects. Statistical significance was set at $\mathrm{P}<0.05$.

\section{RESULTS}

\section{Epidermal thickness}

We found a statistically significant difference in epidermal thickness measurements between subjects $(\mathrm{P}<0.05)$. Epidermal thickness represented $2.5 \%$ to $9.9 \%$ of the overall skin thickness.

In addition, when different regions of the thigh were compared, there was a significant difference in epidermal thickness between regions $(\mathrm{P}<0.05)$. The anterolateral thigh region had the highest mean epidermal thickness and the most consistent epidermal thickness $(60 \pm 3.2 \mu \mathrm{m})$ between subjects (Table 2).

\section{Overall skin thickness}

Similarly, there was a statistically significant difference in overall skin thickness between subjects $(\mathrm{P}<0.05)$. The overall skin thickness was measured as $1,032 \pm 435 \mu \mathrm{m}$ in the anteromedial region compared to $1,220 \pm 257 \mu \mathrm{m}$ in the anterolateral region (Table 2).

In five of the six subjects, there were statistically significant dif-
Fig. 5. Overall skin thickness (combined epidermal and dermal)

In the majority of cases (except C6), there were statistically significant differences in skin thickness measurements (mean \pm standard deviation) when different regions of the thigh (anteromedial [AM], anterior [ANT] and anterolateral $[\mathrm{AL}])$ were compared $(\mathrm{P}<0.05)$.

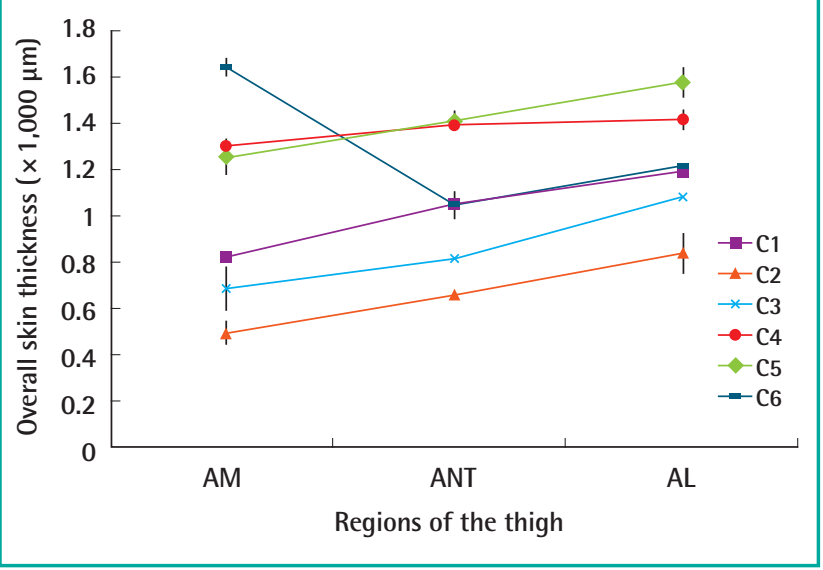

ferences in skin thickness measurements $(\mathrm{P}<0.05)$ when different regions of the thigh were compared. Overall skin thickness increased as we moved laterally from the anteromedial region of the thigh to the anterior and anterolateral regions (Fig. 5).

\section{DISCUSSION}

The choice of a split-thickness skin graft donor site can be influ- 
enced by the surgeon's preference or the patient's choice (Table 1). In addition, the defect size, technical limitations, potential morbidity, patient position, and ease of access to the donor site can also be relevant. While these considerations may be less important when resurfacing large surface areas, small- to moderatesized wounds deserve a balanced debate about which donor site will optimize healing.

In this study, we compared the skin thickness in the anteromedial, anterior, and anterolateral aspects of the thigh, as they are the most common donor sites in clinical practice. The skin of the ventral, outer, and dorsal aspects of the thigh has been described as of intermediate thickness for the purposes of skin grafting [15]. The anterior and anterolateral aspects of the thigh are usually favoured because of their flat surfaces, while the anteromedial aspect may be preferred because of its relatively concealed location.

To optimize measurement accuracy, we developed a method to prepare skin sections using a custom-made apparatus. Random point selection using a grid was employed to eliminate observer bias when measuring epidermal thickness. Following these protocols to overcome potential sampling inaccuracy and observer bias, we found that the anterolateral thigh region was the optimal donor site. Despite subject-to-subject variation, the anterolateral thigh exhibited highest epidermal and overall skin thickness; moreover, the epidermal thickness measurements were relatively consistent across subjects.

We recognize that the number of cadavers used in this study was small and the use of fixed cadaveric skin specimens has the theoretical drawback of being susceptible to potential shrinkage. Although some controversy exists regarding the extent of shrinkage, previous published work dealing with tissue fixation can give an indication of the expected degree of tissue shrinkage. Using liver tissue, Fox et al. showed that a mere 3\% of shrinkage occurred when tissue samples were fixed at room temperature, but that shrinkage was negligible when fixed at $37^{\circ} \mathrm{C}$ [16]. Cutts [17] reported no significant loss in muscle length when muscle tissues were fixed in situ on the skeleton, compared with a small loss $(2 \%)$ in length when fixed in isolation.

Data on the shrinkage of cutaneous tissue following tissue fixation are less conclusive. Hudson-Peacock et al. [18] demonstrated that skin specimens shrank by $31 \%$ after fixation, with most of the shrinkage (22\%) occurring during the post-excision/pre-fixation phase. However, Golomb et al. [19] showed that $94 \%$ of shrinkage occurred after excision and before formalin fixation. Contrastingly, Gregory et al. [20] reported a $22 \%$ shrinkage of skin specimens with equal contributions from both post-excisional/pre-fixation shrinkage and post-fixation shrinkage, but their study size was small. Interestingly, Kerns et al. [21] reported that skin excision specimens shrunk immediately after excision due to their intrinsic contractile properties but re-expanded slightly when immersed in formalin. Another recent report by Dauendorffer et al. [22] similarly showed that shrinkage of skin specimens occurred immediately after excision but the specimens did not show significant changes following fixation. Thus, extrapolating from these reports, we could infer that the fixation-related shrinkage of skin specimens varies between $0 \%$ to a maximum of $10 \%$. We believe that if this study were to be repeated in a clinical setting, the majority of shrinkage would occur immediately following excision, due to primary skin contraction, and before specimen preparation. Therefore, we believe that our present findings would not be markedly different from that of a study using fresh tissue.

Interestingly, a recent survey of the general female population showed a preference for posterior donor sites in the buttock, lower back, and thigh [23]. A previous study also found that the buttock was the donor site of choice amongst surgeons if they were to undergo skin grafting themselves [24]. In paediatric patients with burns, although there were no differences one year after grafting, posterior donor sites had a better cosmetic appearance compared to thigh donor sites at one year after grafting or earlier [25]. Future studies including the buttock, lower back, and the posterior thigh should be carried out to evaluate skin thickness at these sites. Furthermore, measurements of freshly frozen cadaveric tissues or clinical specimens could be performed to confirm our observations.

Our cadaveric study confirms that the anterolateral thigh is the optimal site for harvesting split-thickness skin grafts. Based on skin thickness measurements, the anterolateral thigh has the thickest epidermal and dermal layers. We suggest that the anterolateral thigh region should be the donor site of choice for split-skin graft harvests from the thigh.

\section{REFERENCES}

1. Atiyeh BS, Al-Amm CA, Nasser AA. Improved healing of split thickness skin graft donor sites. J Appl Res 2002;2:6-9.

2. Batkin C, Li F, Maitz P. Hypertrophic scarring of skin graft donor sites in burns patients: prevalence and risk factors [Internet]. Albany Creek: Australian and New Zealand Burn Association; 2013 [cited 2014 Oct 18]. Available from: http:// www.cdesign.com.au/anzba2013/posters/anzba2013asm1final00094.pdf.

3. Dornseifer U, Lonic D, Gerstung TI, et al. The ideal splitthickness skin graft donor-site dressing: a clinical comparative trial of a modified polyurethane dressing and aquacel. Plast Reconstr Surg 2011;128:918-24. 
4. O’Donoghue JM, O’Sullivan ST, Beausang ES, et al. Calcium alginate dressings promote healing of split skin graft donor sites. Acta Chir Plast 1997;39:53-5.

5. Vaingankar NV, Sylaidis P, Eagling V, et al. Comparison of hydrocellular foam and calcium alginate in the healing and comfort of split-thickness skin-graft donor sites. J Wound Care 2001;10:289-91.

6. Bettinger D, Gore D, Humphries Y. Evaluation of calcium alginate for skin graft donor sites. J Burn Care Rehabil 1995; 16:59-61.

7. Giele H, Tong A, Huddleston S. Adhesive retention dressings are more comfortable than alginate dressings on split skin graft donor sites--a randomised controlled trial. Ann R Coll Surg Engl 2001;83:431-4.

8. Hormbrey E, Pandya A, Giele H. Adhesive retention dressings are more comfortable than alginate dressings on splitskin-graft donor sites. Br J Plast Surg 2003;56:498-503.

9. Chuenkongkaew T. Modification of split-thickness skin graft: cosmetic donor site and better recipient site. Ann Plast Surg 2003;50:212-4.

10. Weyandt GH, Bauer B, Berens N, et al. Split-skin grafting from the scalp: the hidden advantage. Dermatol Surg 2009; 35:1873-9.

11. Khalid K, Tarar MN, Mahmood F, et al. Scalp as a donor site for split thickness skin grafts. J Ayub Med Coll Abbottabad 2008;20:66-9.

12. Robert N, May P, Binder JP, et al. The scalp as a donor site for split-thickness skin graft: a rare complication case report. J Plast Reconstr Aesthet Surg 2011;64:e118-20.

13. Winter GD. Formation of the scab and the rate of epithelization of superficial wounds in the skin of the young domestic pig. Nature 1962;193:293-4.

14. Jensen EB, Gundersen HJ, Osterby R. Determination of membrane thickness distribution from orthogonal intercepts. J Microsc 1979;115:19-33.

15. Ostrovskiy NV. Selection of the skin graft thickness with regard to structure of the donor site skin. Acta Chir Plast 1985; 27:145-51.

16. Fox $\mathrm{CH}$, Johnson FB, Whiting J, et al. Formaldehyde fixation. J Histochem Cytochem 1985;33:845-53.

17. Cutts A. Shrinkage of muscle fibres during the fixation of cadaveric tissue. J Anat 1988;160:75-8.

18. Hudson-Peacock MJ, Matthews JN, Lawrence CM. Relation between size of skin excision, wound, and specimen. J Am Acad Dermatol 1995;32:1010-5.

19. Golomb FM, Doyle JP, Grin CM, et al. Determination of preexcision surgical margins of melanomas from fixed-tissue specimens. Plast Reconstr Surg 1991;88:804-9.

20. Gregory N, Mulvaney M, Pattison T, et al. Shrinkage of skin excision specimens and downcoding. Arch Dermatol 2003; 139:542-3.

21. Kerns MJ, Darst MA, Olsen TG, et al. Shrinkage of cutaneous specimens: formalin or other factors involved? J Cutan Pathol 2008;35:1093-6.

22. Dauendorffer JN, Bastuji-Garin S, Guero S, et al. Shrinkage of skin excision specimens: formalin fixation is not the culprit. Br J Dermatol 2009; 160:810-4.

23. Garcia E, Stone E, Chan LS, et al. Donor-site preferences in women during autologous skin grafting. Plast Reconstr Surg 2014;133:378e-382e.

24. White N, Hettiaratchy S, Papini RP. The choice of splitthickness skin graft donor site: patients' and surgeons' preferences. Plast Reconstr Surg 2003;112:933-4.

25. Greenhalgh DG, Barthel PP, Warden GD. Comparison of back versus thigh donor sites in pediatric patients with burns. J Burn Care Rehabil 1993;14:21-5. 